therapy ( $1 \mathrm{gr} / \mathrm{kg}$ in three devided doses) was administered each month for six months.

Marked improvement in dermal eruptions was observed following the second administration of IVIG therapy. Now in the eighth month of follow up she has been complaining of dyspnea on exertion for twenty days. High resolution computerised tomography (HRCT) of lung revealed paranchimal changes suggesting that bronchiolitis obliterans organising pneumonia (BOOP).

Could this be the result of methotrexate therapy or a result of amyopathic dermatomyositis ? Lung involvement in amyopathic dermatomyositis is rare. Pulmonary drug toxicity is increasingly being diagnosed as a cause of acute and chronic lung disease. BOOP, which is commonly caused by cyclophosphamide and bleomycin as well as methotrexate and gold salts, appears on HRCT scans as poorly defined nodular areas of consolidation, centrilobular nodules, and bronchial dilatation. Knowledge of the drugs most frequently involved can facilitate diagnosis and institution of appropriate treatment. The results of lung biopy will be discussed in poster session. If lung biopsy will reveal the pathology methotrexate will be discontinued.

Objectives

Methods

Results

Conclusion

\section{AB0219 QUANTITATIVE HEMOSTATIC INDICES IN SECONDARY AND MYELOPROLIFERATIVE THROMBOCYTOSIS}

1J Parada-Turska, ${ }^{2}$ B Sokolowska, ${ }^{2}$ A Walter-Croneck, ${ }^{2}$ A Dmoszynska, 'L Szczepanski, 2J Brzozowska, ${ }^{2} \mathrm{M}$ Sledzinska, ${ }^{2} \mathrm{M}$ Kokoszka. ${ }^{1}$ Department of Rheumatology; ${ }^{2}$ Department of Hematology, Medical University, Lublin, Poland

\subsection{6/annrheumdis-2001.740}

Background An elevated platelet count may lead to an increased incidence of systemic thromboembolism or unexpectedly to thrombohemorragic complications.

Both, incidences of systemic thromboembolism and unexpected thrombohemorragic complications (bleeding) were described in patients with an elevated platelet count.

In the present study the quantitative hemostatic indices were measured in 46 patients with thrombocytosis (platelet count > 400.000 per microliter), including 23 patients with secondary thrombocytosis (17 patients with rheumatoid arthritis, 2 with lung cancer, 1 with systemic lupus erythematosus, 1 with granuloma Wegeneri) and 23 patients with myeloproliferative thrombocytosis (12 with chronic myeloid leukaemia, 8 with polycythemia vera, 3 with idiopathic thrombocytosis).

In both studied groups, von Willebrand factor activity and epinephrine-induced platelet aggregation were decreased, and activated partial thromboplastin time was prolonged, thus, suggesting increased risk of bleeding complications.

On the other hand, the concentrations of fibrinogen and $\mathrm{d}$ dimmers were enhanced and bleeding time was shortened. These findings argue for an enhanced risk of thrombosis.

Since no differences between two studied groups were found, it seems that the observed abnormalities were directly related to the elevated platelet count and were not associated with primary disease.

The low incidence of hemostatic complications in patients with secondary and myeloproliferative thrombosis may suggest that the coexistence of an additional component is critical for the clinical manifestation of such disturbances.
Objectives

Methods

Results

Conclusion

\section{AB0220 OCHRONOTIC SPONDYLOARTHROPATHY WITH MULTISYSTEMIC INVOLVEMENT: A CASE REPORT}

K Nas, A Gür, R Çevik, AJ Saraç, S Söker (Çakmak), S Em, M Karakoç, S Kilinç. Physical Medicine and Rehabilitation, Dicle University, Diyarbakir, Turkey

\subsection{6/annrheumdis-2001.741}

Background Ochronosis is a musculoskeletal manifestation of alcaptonuria, a rare hereditery metabolic disorder characterised by the absence of the enzyme homogentisic acid oxidase and associated with various systemic abnormalites related to the deposition of homogentisic acid pigment.

Objectives We aimed to review a case with ochronosis, a disorder of rare and multisystemic involvement, in the light of literature.

Methods A housewife, 53 years of age, was admitted to our clinic with complaints of low back and left shoulder pains. Due to low-back pain complaints, she was operated on two times with diagnosis of lumbar discal hernia.

Results The radiographic appearance in the lumbar spine of the patients was almost pathogonomic of ochronotic spondyloartropathy. Thypical water-like calcifications were seen in the intervertebral discs, with narrowing of disc spaces and osteoporotic rarefaction of the vertebral bodies. Shoulder joint, narrowing of the joint space, a marked subchondral sclerosis, and small osteophytes were observed; similarly, knee, sacroiliac joints and symphysis pubis show at narrowing of the joint spaces and subchondral bone sclerosis.

In the eyes of the patient, ochronotic pigmentation was seen by light microscopy. The blue-black pigmentation was bilateral and asymmetric in the cornea, conjunctiva and sclera. In addition, black-grey pigmentation was present in the ear cartilage and hand nails. In the laboratory test, homogentisic acid was found increase $(2740 \mathrm{mmol} \mathrm{HjA} / \mathrm{mmol}$ creatinin; $\mathrm{N}$ : $10 \mathrm{mmol}$ $\mathrm{HjA} / \mathrm{mmol}$ ) creatinin in urine.

Conclusion

\section{AB0221 FAMILIAL MEDITERRANEAN FEVER: ANALYSIS OF 45 TUNISIAN PATIENTS}

SS Turki, CH Ben Taarit, R Goucha, E Abderrahim, F Ben Moussa, H Ben Maiz. Department of Nephrology and Internal Medicine, Charles Nicolle Hospital, Tunis, Tunisia

\subsection{6/annrheumdis-2001.742}

Background Familial mediterranean fever (FMF) is an inherited multisystem disease of unknown aetiology, characterised by recurrent, painful, self-limited episodes.

Objectives The objectives of this study is to describe clinical profile, course and complications of 45 cases of FMF observed over a period of 25 years.

Methods Retrospective review of 45 cases with FMF.

Results Forty patients (88\%) started their illness below the age of 10 years. Peritonitis occured in $84,4 \%$, arthritis in $35,5 \%$, pleuretis in $17,7 \%$ and erysipelas-like lesions in $31,1 \% .21$ patients developed renal amyloïdosis and 17 patients were subjected to unnecessary operative surgery. 